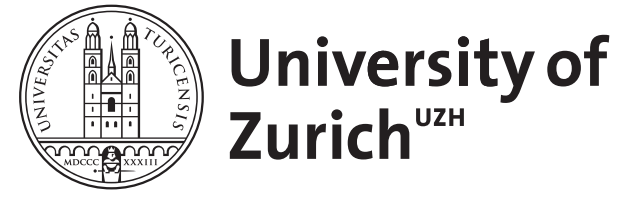

\title{
Hiding robot inertia using resonance
}

\author{
Vallery, H ; Duschau-Wicke, A ; Riener, R
}

\begin{abstract}
To enable compliant training modes with a rehabilitation robot, an important prerequisite is that any undesired human-robot interaction forces caused by robot dynamics must be avoided, either by an appropriate mechanical design or by compensating control strategies. Our recently proposed control scheme of "Generalized Elasticities" employs potential fields to compensate for robot dynamics, including inertia, beyond what can be done using closed-loop force control. In this paper, we give a simple mechanical equivalent using the example of the gait rehabilitation robot Lokomat. The robot consists of an exoskeleton that is attached to a frame around the patient's pelvis. This frame is suspended by a springloaded parallelogram structure. The mechanism allows vertical displacement while providing almost constant robot gravity compensation. However, inertia of the device when the patient's pelvis moves up and down remains a source of large interaction forces, which are reflected in increased ground reaction forces. Here, we investigate an alternative suspension: To hide not only gravity, but also robot inertia during vertical pelvis motion, we suspend the robot frame by a stiff linear spring that allows the robot to oscillate vertically at an eigenfrequency close to the natural gait frequency. This mechanism reduces human-robot interaction forces, which is demonstrated in pilot experimental results.
\end{abstract}

DOI: https://doi.org/10.1109/IEMBS.2010.5626416

Other titles: 32nd Annual International Conference of the IEEE EMBS Buenos Aires, Argentina, August 31 - September 4, 2010

Posted at the Zurich Open Repository and Archive, University of Zurich ZORA URL: https://doi.org/10.5167/uzh-43964

Journal Article

Originally published at:

Vallery, H; Duschau-Wicke, A; Riener, R (2010). Hiding robot inertia using resonance. IEEE Engineering in Medicine and Biology Society. Conference Proceedings, 1:1271-1274.

DOI: https://doi.org/10.1109/IEMBS.2010.5626416 


\title{
Hiding Robot Inertia Using Resonance
}

\author{
Heike Vallery ${ }^{1,2}$, Alexander Duschau-Wicke ${ }^{1,2,3}$, and Robert Riener ${ }^{1,2}$ \\ ${ }^{1}$ Sensory-Motor Systems (SMS) Lab, Institute of Robotics and Intelligent Systems (IRIS), ETH Zurich \\ ${ }^{2}$ Medical Faculty, Balgrist University Hospital, University of Zurich, Switzerland \\ ${ }^{3}$ Hocoma AG, Volketswil, Switzerland.
}

\begin{abstract}
To enable compliant training modes with a rehabilitation robot, an important prerequisite is that any undesired human-robot interaction forces caused by robot dynamics must be avoided, either by an appropriate mechanical design or by compensating control strategies. Our recently proposed control scheme of "Generalized Elasticities" employs potential fields to compensate for robot dynamics, including inertia, beyond what can be done using closed-loop force control. In this paper, we give a simple mechanical equivalent using the example of the gait rehabilitation robot Lokomat. The robot consists of an exoskeleton that is attached to a frame around the patient's pelvis. This frame is suspended by a springloaded parallelogram structure. The mechanism allows vertical displacement while providing almost constant robot gravity compensation. However, inertia of the device when the patient's pelvis moves up and down remains a source of large interaction forces, which are reflected in increased ground reaction forces. Here, we investigate an alternative suspension: To hide not only gravity, but also robot inertia during vertical pelvis motion, we suspend the robot frame by a stiff linear spring that allows the robot to oscillate vertically at an eigenfrequency close to the natural gait frequency. This mechanism reduces human-robot interaction forces, which is demonstrated in pilot experimental results.
\end{abstract}

\section{INTRODUCTION}

Recent multicenter controlled trials showed that subacute and chronic stroke patients still profit more from conventional manual therapy than from robotic gait training, at least when the robot imposes movements via position control along a fixed reference trajectory [1], [2]. New results on motor learning and neural plasticity can help explain this by the fact that position control does not allow the human to influence the gait pattern and to make errors, which is necessary for learning and the formation of an internal task representation [3], [4]. Furthermore, the robot does not require active participation of the patient, which is a key element for recovery [5]-[8]. These results encourage socalled patient-cooperative control of rehabilitation robots, which allows the human to actively influence the gait pattern and to make errors.

To achieve this, any undesired interaction forces between robot and human (due to inherent robot dynamics) should be minimized, meaning that the robot must be transparent. Forces that need to be overcome when moving a robot are inertia, gravity, Coriolis and centrifugal forces, and friction. A lightweight construction and/or compliant actuation [9], [10] reduce these forces, but this reduction is limited, especially when the robot is a versatile device that also needs to stiffly guide severely affected patients. Besides hardware design, there are also control strategies available to reduce apparent robot dynamics. However, these strategies cannot fully compensate the robot, where the main problem is generally to hide inertia. The most prominent strategy to reduce inertia is force feedback, realized via admittance or impedance control concepts [11], [12]. However, due to stability limits, the user will always feel some residual apparent inertia [13].

In gait rehabilitation robots like the Lokomat [14], [15], robot inertia is highly relevant: Human gait is a very dynamic, even ballistic motion, such that the robot's inertia causes high undesired interaction forces acting on the human's legs. The common strategy in robot control would be to tolerate this remaining inertia and to compensate "at least" the other force components, especially robot gravity. However, we have shown that gravity compensation of the robot is not always an effective means to reduce interaction forces [16]. On the contrary, gravity compensation of leg exoskeletons during gait is even counterproductive, and it increases interaction forces. This can be explained by the natural dynamics of the exoskeleton legs, which resemble those of a pendulum during the swing phase: They swing easily with gravity helping to accelerate and decelerate the inert mass. As robot and human leg are mechanically similar, they swing almost in parallel to each other, and only little interaction forces are exchanged between them. Without gravity acting on the exoskeleton leg, accelerating and decelerating forces have to be exerted on the robot by the human to overcome the exoskeleton's inertia, which severely increases interaction forces between human and robot. Inspired by this observation that the earth's gravitational field partially compensates robot inertia during gait, we have recently proposed the concept of Generalized Elasticities as a generic tool to hide robot dynamics using potential fields [16]. Given a particular robot and an estimate of the type of motion a human operator will perform (like walking in a gait rehabilitation robot), the optimal potential field manipulates robot dynamics in such a way that the resulting interaction forces between robot and operator are minimized.

A similar effect as in the exoskeleton legs can be observed for the vertical frame motion of stationary gait rehabilitation robots like the Lokomat or the LOPES, which are attached to the patient's pelvis. The conventional procedure is to compensate gravity of the frame and the attached exoskele- 
ton legs. In the LOPES gait rehabilitation robot, a springloaded parallelogram mechanism provides a constant gravitycounteracting force [17], and in the Lokomat, a pre-loaded compliant spring has a very similar effect. The drawback of this type of attachment is that inertial forces are not compensated for, and vertical displacement of the patient's center of mass leads to considerable interaction forces with the robot. In the Lokomat, a mechanism to overcome these inertial forces has been investigated several years ago, where the originally passive vertical DoF was equipped with an actuator [18]. This actuator allowed to move the frame up and down synchronously with the exoskeleton legs. However, this only worked when patients were rigidly guided by the device, as the trajectory had to be known in advance. Furthermore, any actuator further increases system inertia and complexity.

For this problem, the concept of Generalized Elasticities can be translated to a simple solution in the hardware domain: When a spring of appropriate stiffness supports the robot frame, the vertical motion that the user performs becomes the "eigenmotion" of the robot. This means that the robot requires only very low forces from the human in order to "oscillate" up and down in the frequency of gait, parallel to the human. This oscillation is highly compliant, meaning that it is not intended to guide, support, or even force the human onto an expected trajectory. The robot only optimally compensates its own dynamics on this trajectory. Deviations from the eigenmotion of the robot should not lead to a large increase in interaction forces; the compensation might only not be optimal anymore (for example in case of a deviating step frequency). This very simple idea is investigated in this paper in terms of robustness to variations in the shape of the pelvis motion and/or the step frequency.

Preliminary results with healthy subjects walking in the Lokomat gait rehabilitation robot are presented.

\section{DYNAMICS SHAPING VERSUS GRAVITY CANCELlation}

\section{A. Optimal Robot Suspension Design}

In order to relieve patients undergoing gait rehabilitation from the robot's weight without constraining vertical pelvis motion, there is commonly a support mechanism that cancels robot gravity by a constant (or almost constant) vertical force (Fig. 1, left). Alternative to this conventional concept, we investigate a spring suspension, aiming to compensate both for gravity and inertia of the robot (Fig. 1, right).

For a gravity-canceling mechanism (case 1), the support force is constant:

$$
F_{\text {support,const }}=m_{\text {rob }} g,
$$

with $m_{\text {rob }}$ being the robot mass that moves vertically. In the investigated spring suspension (case 2), the force is proportional to vertical displacement, where the spring offset is chosen such that it cancels gravity:

$$
F_{\text {support,spring }}=m_{\mathrm{rob}} g-c_{s} z \text {. }
$$

The spring stiffness is now tuned so that the resulting eigenfrequency of the robot approaches the frequency $\omega_{0}$ of

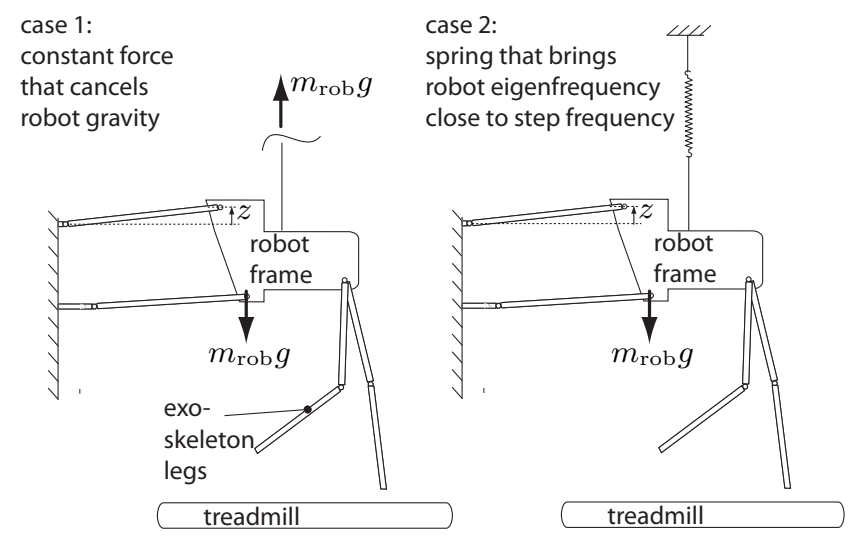

Fig. 1. Suspension cases: The commonly employed constant-force support cancels robot gravity. In contrast, the spring support gives the robot an eigenmotion close to human pelvis motion during gait, in order to hide both robot gravity and inertia.

vertical pelvis displacement in human gait. To achieve this, the spring stiffness $c_{s}$ should be

$$
c_{s}=m_{\mathrm{rob}} \omega_{0}^{2}
$$

The interaction forces $F_{\text {int }}$ between robot and human that are needed to move the entire robot up and down are in both cases:

$$
F_{\text {int }}=m_{\text {rob }}(g+\ddot{z})-F_{\text {support }} .
$$

For a constant gravity-canceling support, this interaction force is

$$
F_{\text {int,const }}=m_{\text {rob }} \ddot{z},
$$

i.e. equal to the entire inertial force caused by the robot. For the investigated spring suspension, the force is

$$
F_{\text {int,spring }}=m_{\text {rob }}\left(\ddot{z}+\omega_{0}^{2} z\right) .
$$

In case of sinusoidal motion with the frequency according to (3), $\ddot{z}$ cancels $\omega_{0}^{2} z$, so interaction forces would be zero, regardless of the motion amplitude.

\section{B. Sensitivity to Imperfect Choice of Spring Characteristics}

For now, only the case of sinusoidal pelvis motion with matching frequency has been investigated. For a practical application, it is important to know how robustly the compensation is achieved for pelvis motion that deviates from the sinusoidal assumption and that also exhibits a frequency that differs from the robot's eigenfrequency in vertical direction. These two aspects will now be analyzed more closely.

1) Deviating step frequency: In case the human walks with an angular frequency $\tilde{\omega}_{0}$ deviating from the chosen eigenfrequency $\omega_{0}$ of the robot, the acceleration is

$$
\ddot{z}=-\tilde{\omega}_{0}^{2} z,
$$

such that the interaction force of (6) is:

$$
F_{\text {int }}=m_{\mathrm{rob}} z\left(\omega_{0}^{2}-\tilde{\omega}_{0}^{2}\right) .
$$

Compared to the constant-force support, where the interaction force is $-m_{\mathrm{rob}} z \tilde{\omega}_{0}^{2}$, the resulting force magnitude 
is considerably lower even for large deviations from the expected frequency. Only for robot eigenfrequencies that exceed the gait frequency by a factor of $\sqrt{2}$ (meaning in return that the stiffness of the spring has been chosen erroneously to be more than double the required value), interaction forces could increase. That means that as long as the spring stiffness is larger than zero and smaller than twice the optimal stiffness, any spring support is better than the constant-force suspension. Therefore, the support is very robust to changes in step frequency.

2) Not sinusoidal pelvis motion: In case of not sinusoidal motion, there are higher harmonics in the pelvis translation profile, such that the displacement $z$ can be expressed as:

$$
z=\sum_{k=1}^{n} a_{k} \sin \left(k \omega_{0} t-\varphi_{k}\right) .
$$

With the acceleration

$$
\ddot{z}=-\omega_{0}^{2} \sum_{k=1}^{n} k^{2} a_{k} \sin \left(k \omega_{0} t-\varphi_{k}\right),
$$

the interaction force of $(6)$ is

$$
F_{\text {int }}=m_{\text {rob }} \omega_{0}^{2} \sum_{k=1}^{n}\left[\left(1-k^{2}\right) a_{k} \sin \left(k \omega_{0} t-\varphi_{k}\right)\right] .
$$

As $\left|1-k^{2}\right|<k^{2} \forall k \geq 1$, the amplitude of each harmonic gets reduced, although not very efficiently for the higher harmonics. To further improve behavior for a strongly nonsinusoidal signal, a nonlinear spring would be even better. Nevertheless, the linear spring is still superior to the constantforce suspension, as it reduces at least the first harmonics (which generally have the highest amplitudes) as opposed to none.

\section{Experimental Design}

Practical experiments with healthy subjects were conducted with the Lokomat gait rehabilitation robot, which has a kinematic structure as depicted in Fig. 1. Normally, the robot's weight is compensated by a heavily pre-loaded support spring attached within the parallelogram structure. This spring is subjected only to small deformations, which leads to a very small reflected stiffness with respect to vertical frame motion. Thus, the mechanism provides an almost constant support force to cancel robot gravity, as in the theoretical case 1 in Fig. 1.

To allow comparable experimental conditions, the original spring within the parallelogram was unfastened, and the two support cases were emulated using the Lokolift weight support system [19]. Normally used to partially compensate a patient's body weight, the Lokolift was now attached to the robot directly (the subjects wore no harness), so that the robot was suspended by the Lokolift. The Lokolift disposes of a force sensor to measure rope tension, and this sensor was used for force control. A potentiometer was integrated in the robot's parallelogram mechanism to measure vertical displacement $z$.
Two different control conditions were implemented according to the two investigated cases: In condition 1, the support force $F_{\text {support }}$ was set constant, so that the Lokolift emulated gravity cancellation, rendering the force as in (1). In condition 2, the support force was set to imitate a direct spring suspension, i.e. proportional to vertical displacement as in (2). The offset of this vertical spring was chosen identical to the constant force in the previous condition. The spring stiffness was tuned so that the resulting eigenfrequency of the robot approached the frequency of vertical pelvis displacement in human gait of approximately $1.5 \mathrm{~Hz}$. As shown in the last section, exact matching is not necessary. The stiffness was chosen before the experiments, and it was not adjusted to the individual subjects nor to treadmill speed. The exoskeleton legs were always controlled to zero interaction torques with the subject, using the concept of Generalized Elasticities, as described in [16].

Three healthy subjects (S1: f, $64 \mathrm{~kg}, \mathrm{~S} 2: \mathrm{m}, 89 \mathrm{~kg}, \mathrm{~S} 3: \mathrm{f}$, $54 \mathrm{~kg}$ ) took part in the study. Each subject walked once on the treadmill without the robot, once with the robot and constant suspension force, and once with the robot and the emulated spring suspension. Conditions in the robot were randomized and lasted approximately two minutes each. Two treadmill speeds were tested, 2 and $3 \mathrm{~km} / \mathrm{h}$. During these experiments, the net vertical ground reaction force was recorded from force plates in the treadmill.

The net ground reaction force $F_{\mathrm{GR}}$ reflect weight of the human $\left(m_{\mathrm{hum}} g\right)$, weight of the robot, and inertial forces, as far as these forces are not compensated by the support force:

$$
F_{\mathrm{GR}}=\left(m_{\text {hum }}+m_{\text {rob }}\right)(g+\ddot{z})-F_{\text {support }} .
$$

This ground reaction force can be decomposed into a part $F_{\mathrm{GR} \text {,free }}$ that would, for the same motion, also be present on the treadmill without the robot, and the interaction force $F_{\text {int }}$ with the robot:

$$
F_{\mathrm{GR}}=m_{\mathrm{hum}}(g+\ddot{z})+F_{\mathrm{int}}=F_{\mathrm{GR}, \text { free }}+F_{\mathrm{int}} .
$$

If the robot was fully transparent, ground reaction forces should be identical to free gait on the treadmill.

The amount of variation in ground reaction forces reflects the dynamic forces caused by inertia, both of the subject and of the robot. Therefore, the standard deviation of ground reaction forces in comparison to free gait on the treadmill is chosen as a measure of uncompensated robot inertia. In order to remove transient effects, only the data recorded within the last 30 steps of each condition were used for analysis.

\section{RESUlts AND Discussion}

In direct comparison with constant gravity compensation (condition 1), the spring suspension showed to be more effective in terms of transparency for healthy subjects walking in the robot. The effect increases with gait speed, and it seems to be more pronounced for lighter subjects.

This is of course based on data of only three pilot subjects, and human adaptation over the course of the experiment has not been analyzed. Further investigations will have to address the question whether reduced interaction forces also 

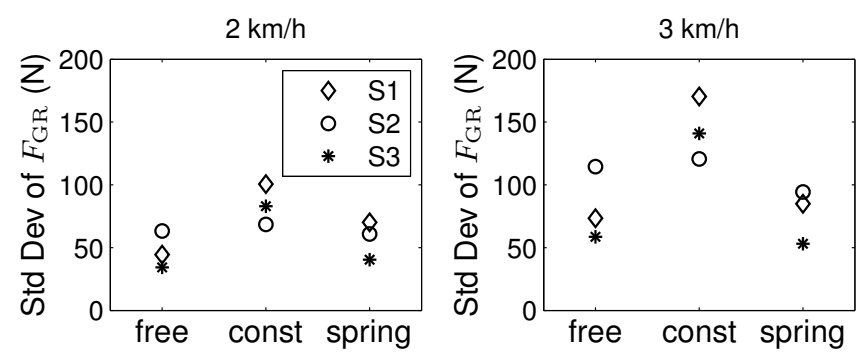

Fig. 2. Standard deviations of ground reaction forces of three healthy subjects walking freely on the treadmill (free), with the robot and constantforce robot suspension (const), and with the robot and spring suspension (spring).

lead to a reduction of undesired adaptation to the robot: In order to reduce inertial forces when walking with the constant-force robot support, subjects might reduce their movement amplitude or their cadence compared to free gait, and this undesired effect may be alleviated with the spring compensation. We had observed a similar behavior for the exoskeleton legs [16]. In this context, we will also look at whether subjects tend to synchronize their cadence with the robot's eigenfrequency.

For a practical realization, the spring support does not need to be overhead; the same behavior can e.g. for the Lokomat be achieved by exchanging the current spring in the parallelogram mechanism, simply increasing the reflected stiffness.

\section{CONCLUSION}

The results show that optimizing passive behavior of a rehabilitation robot can be effective to enhance transparency, and this procedure is superior to pure gravity cancellation. Preliminary results with the Lokomat indicate that robot inertia and gravity can be hidden simultaneously by very simple mechanical design considerations. Eventually, less undesired interaction forces allow more compliant training modes with active patient participation, potentially improving rehabilitation outcome.

\section{ACKNOWLEDGMENTS}

The contents of this publication were developed in part with support of the National Center of Competence in Research (NCCR) on Neural Plasticity and Repair of the Swiss National Science Foundation and in part under a grant from the US Department of Education, NIDRR grant number H133E070013. However, those contents do not necessarily represent the policy of the US Department of Education, and you should not assume endorsement by the Federal Government of the United States of America.

\section{REFERENCES}

[1] T. G. Hornby, D. D. Campbell, J. H. Kahn, T. Demott, J. L. Moore, and H. R. Roth, "Enhanced gait-related improvements after therapistversus robotic-assisted locomotor training in subjects with chronic stroke: a randomized controlled study." Stroke, vol. 39, no. 6, pp. 1786-1792, Jun 2008.
[2] J. Hidler, D. Nichols, M. Pelliccio, K. Brady, D. D. Campbell, J. H. Kahn, and T. G. Hornby, "Multicenter randomized clinical trial evaluating the effectiveness of the lokomat in subacute stroke." Neurorehabilitation and Neural Repair, vol. 23, no. 1, pp. 5-13, 2009.

[3] R. Shadmehr and F. A. Mussa-Ivaldi, "Adaptive representation of dynamics during learning of a motor task," Journal of Neuroscience, vol. 14, pp. 3208-24, 1994.

[4] R. A. Scheidt, J. B. Dingwell, and F. A. Mussa-Ivaldi, "Learning to move amid uncertainty," Journal of Neurophysiology, vol. 86, pp. 97185, 2001.

[5] E. Taub, G. Uswatte, and R. Pidikiti, "Constraint-induced movement therapy: a new family of techniques with broad application to physical rehabilitation: a clinical review," Journal of Rehabilitation Research \& Development, vol. 36, no. 3, pp. 237-51, 1999.

[6] L. L. Cai, A. J. Fong, C. K. Otoshi, Y. Liang, J. W. Burdick, R. R. Roy, and V. R. Edgerton, "Implications of assist-as-needed robotic step training after a complete spinal cord injury on intrinsic strategies of motor learning," Journal of Neuroscience, vol. 26, no. 41, pp. 10564 8, 2006.

[7] N. Hogan, H. I. Krebs, B. Rohrer, J. J. Palazzolo, L. Dipietro, S. E. Fasoli, J. Stein, R. Hughes, W. R. Frontera, D. Lynch, and B. T. Volpe, "Motions or muscles?: Some behavioral factors underlying robotic assistance of motor recovery," Journal of Rehabilitation Research \& Development, vol. 43, no. 5, pp. 605-18, 2006.

[8] H. I. Krebs, J. J. Palazzolo, L. Dipietro, B. T. Volpe, and N. Hogan, "Rehabilitation robotics: Performance-based progressive robot-assisted therapy," Autonomous Robots, vol. 15, pp. 7-20, 2003.

[9] G. A. Pratt, M. M. Williamson, P. Dillworth, J. Pratt, K. Ulland, and A. Wright, "Stiffness Isn't Everything," in International Symposium on Experimental Robotics (ISER), 1995.

[10] H. Vallery, J. Veneman, E. van Asseldonk, R. Ekkelenkamp, M. Buss, and H. van der Kooij, "Compliant Actuation of Rehabilitation Robots - Benefits and Limitations of Series Elastic Actuators," IEEE Robotics and Automation Magazine (RAM), vol. 15, no. 3, pp. 60-69, 2008.

[11] N. Hogan, "Impedance control: An approach to manipulation. Part I - Theory, Part II - Implementation, Part III - Applications," ASME Journal of Dynamic Systems, Measurement, and Control, vol. 107, pp. $1-24,1985$.

[12] B. S. und O. Khatib, Ed., Handbook of Robotics. Springer, 2008.

[13] E. Colgate and N. Hogan, "An analysis of contact instability in terms of passive physical equivalents," in Proceedings of the IEEE International Conference on Robotics and Automation (ICRA), Scottsdale, AZ, USA, 1989, pp. 404-409.

[14] G. Colombo, M. Wirz, and V. Dietz, "Driven gait orthosis for improvement of locomotor training in paraplegic patients," Spinal Cord, vol. 39, no. 5, pp. 252-255, 2001.

[15] R. Riener, L. Lnenburger, I. C. Maier, G. Colombo, and V. Dietz, "Locomotor training in subjects with sensori-motor deficits: An overview of the robotic gait orthosis lokomat," Journal of Healthcare Engineering, vol. 1, no. 2, pp. 197-216, 2010.

[16] H. Vallery, A. Duschau-Wicke, and R. Riener, "Optimized Passive Dynamics Improve Transparency of Haptic Devices," in Proceedings of the IEEE International Conference on Robotics and Automation (ICRA), Kobe, Japan, 2009.

[17] J. F. Veneman, R. Kruidhof, E. E. G. Hekman, R. Ekkelenkamp, E. H. F. Van Asseldonk, and H. Van der Kooij, "Design and Evaluation, of the LOPES Exoskeleton Robot for Interactive Gait Rehabilitation," IEEE Transactions on Neural Systems and Rehabilitation Engineering, vol. 15, no. 3, pp. 379-386, 2007.

[18] N. Laubacher, "Entwurf und Implementierung einer Trägheitskompensation für die automatisierte Gehorthese Lokomat," IFA, ETH Zürich, Tech. Rep., 2005.

[19] M. Frey, G. Colombo, M. Vaglio, R. Bucher, M. Jorg, and R. Riener, "A novel mechatronic body weight support system," IEEE Transactions on Neural Systems and Rehabilitation Engineering, vol. 14, pp. 311-21, 2006. 